Cahiers $d u$ MONDE RUSSE

\section{Cahiers du monde russe}

Russie - Empire russe - Union soviétique et États indépendants

$51 / 4 \mid 2010$

Sciences humaines et sociales en Russie à l'Âge d'argent

\title{
Martine Godet, La pellicule et les ciseaux
}

\section{Birgit Beumers}

\section{(2) OpenEdition}

1 Journals

Édition électronique

URL : https://journals.openedition.org/monderusse/7412

DOI : $10.4000 /$ monderusse. 7412

ISSN : $1777-5388$

Éditeur

Éditions de l'EHESS

Édition imprimée

Date de publication : 25 novembre 2010

Pagination : 755-757

ISBN : 978-2-7132-2316-7

ISSN : $1252-6576$

Référence électronique

Birgit Beumers, «Martine Godet, La pellicule et les ciseaux », Cahiers du monde russe [En ligne], 51/4 I 2010, mis en ligne le 09 décembre 2011, consulté le 03 septembre 2022. URL : http://

journals.openedition.org/monderusse/7412; DOI : https://doi.org/10.4000/monderusse.7412

Ce document a été généré automatiquement le 3 septembre 2022

Tous droits réservés 


\title{
Martine Godet, La pellicule et les ciseaux
}

\author{
Birgit Beumers
}

\section{RÉFÉRENCE}

Martine GODET, La pellicule et les ciseaux. La censure soviétique dans le cinéma soviétique du Dégel à la perestroïka. Paris : CNRS, 2010, 308 p. + index des films

1 Le livre de Martine Godet sur la censure dans le cinéma soviétique couvre une période peu étudiée, qui va du Dégel à la perestroïka. S'il existe de nombreuses études sur le cinéma de l'époque stalinienne, de la perestroïka ou des années vingt, la période de la "stagnation" reste à découvrir. Dans ce contexte, cet ouvrage pourrait être une contribution importante à l'histoire de la politique culturelle et cinématographique des années soixante et soixante-dix.

2 L'introduction, claire, explique les sources et la méthode. L'auteur s'appuie aussi bien sur les témoignages que sur des comparaisons de projets, films complétés et films distribués. Les deux chapitres de la première partie portent sur les mécanismes de la censure. Le premier situe le contexte de la période étudiée. Il présente le rôle de l'intelligentsia comme avant-garde intellectuelle qui s'oppose aux exigences du régime - et qui formera le mouvement dissident -, et la rupture générationnelle entre pères et fils après la guerre pour caractériser le Dégel et son impact sur la production des films à la fin des années cinquante. Les faits sont exposés d'une manière cohérente et compréhensible, même s'il manque parfois la distance qui permettrait de dégager une conception lucide pour la suite de l'ouvrage. Ainsi, on peut regretter que les raisons expliquant la censure de certains thèmes à certains moments ne soient pas remises dans leur contexte politique mais reprennent les termes, empreints de subjectivité, des témoignages de l'époque. Enfin, ce chapitre offre une étude détaillée du film de Marlen Huciev J'ai vingt ans et l'histoire de sa censure, avec une comparaison éclairante des deux versions du film. 
3 Le second chapitre se concentre sur les mécanismes et les thèmes de la censure, il fait appel à de multiples sources secondaires qui témoignent de la connaissance de l'auteur en littérature multilingue sur son thème de recherche. Les sources d'archives sont assez rares dans cette partie, l'auteur s'appuyant sur Polka, l'important ouvrage de Valerij Fomin ${ }^{1}$, dans lequel sont étudiés les cas de censures sous le régime brejnévien. L'explication des structures de production et de contrôle, basée essentiellement sur des entretiens et non sur des documents officiels, ne suffit pas à mettre en évidence une image précise du contrôle idéologique et administratif sur le cinéma. L'auteur se fonde sur des cas individuels (films, réalisateurs, témoignages) et bien connus, mais on aurait souhaité trouver aussi une analyse des structures de contrôle, une discussion sur la perception du "succès ", y compris commercial, par l'État et le parti, ou encore une étude des films dits populaires.

4 Dans la deuxième partie, Godet réécrit l'histoire du cinéma russe de la période brejnévienne en réintroduisant les films censurés dans les biographies artistiques des réalisateurs Aleksej German, Elem Klimov, Kira Muratova et Andrej Končalovskij. L'exercice n'est pas complètement nouveau et pour l'auteur ce sont souvent des raisons personnelles qui ont poussé les censeurs à agir : la peur du contrôle du parti et le désir de création artistique du côté des bureaucrates. On pourrait sans doute aussi prêter aux censeurs des motifs objectifs, comme le désir sincère de protéger les citoyens des mauvaises influences. Des projets non réalisés sont évoqués, dont on aurait aimé savoir davantage: les biographies des réalisateurs auraient gagné en intérêt si elles avaient inclus une analyse des films réalisés, censurés et non réalisés. On regrette que cette réécriture de l'histoire du cinéma de la "stagnation", étayée essentiellement par des sources déjà publiées, ne constitue pas un complément à celles écrites depuis la perestroïka, et n'offre pas de conclusions nouvelles sur la censure des années 1960 et 1970.

5 Le quatrième chapitre évoque une nouvelle génération dans le cinéma du Dégel. Pour une raison inconnue, il s'appuie sur Mihail Romm que l'auteur présente comme un homme « qui n'est pas un cinéaste de grand talent» (p. 146). Ce commentaire ignore simplement les efforts de Romm comme pédagogue au VGIK et son influence précisément sur la génération du cinéma de la stagnation. Le chapitre juxtapose Mémoires et documents d'archives, sans distinguer leur mérite et leur valeur respectifs: les Mémoires, contrairement à un document, sont basés sur des impressions personnelles d'une époque donnée, à un moment précis, et ne peuvent pas relater des faits de façon totalement objective.

6 La conclusion vient en deux étapes. Godet retrace d'abord l'histoire des films non censurés, sans expliquer le principe retenu pour choisir ces films. Dans la discussion sur La parentèle de Nikita Mihalkov, elle attribue la controverse autour du film à son fond social, alors qu'elle portait surtout sur la représentation du départ des soldats pour l'Afghanistan. Dans la seconde étape, l'auteur produit une analyse succincte des aspects commerciaux et artistiques.

7 La bibliographie aurait pu mentionner Real Images de Josephine Woll, le livre d'Aleksandr Prohorov sur le cinéma de la stagnation, les publications de Martin Dewhirst sur la censure $^{2}$, et les interviews et reportages de Sally Laird dans le magazine Index on Censorship.

8 Dans l'annexe, l'auteur présente des entretiens avec German, Klimov, Rjazanceva et Sadčikov qui ont été menés en 1998. Ces entretiens, bien que datés, sont intéressants en ce qu'ils constituent un témoignage du passé sur la stagnation. 
9 Godet a étudié scrupuleusement la période et l'on regrette d'autant plus qu'elle ait parfois manqué, vis-à-vis des faits présentés, du recul nécessaire qui aurait permis de présenter une thèse plus complète sur le fonctionnement de la censure au cinéma dans le contexte idéologique, culturel et historique. Certaines questions restent ouvertes et appellent de futures recherches : le pourquoi des si nombreux cas de censure à la fin des années soixante; le rôle et l'importance de l'anniversaire de la révolution en 1967 ; les interventions de la censure dans les autres sphères de la création artistique (musique, théâtre, peinture). La censure frappe aussi bien des comédies que des films pour les masses : quelles conclusions tirer sur cette censure qui affecte tant les films d'auteur que les films « commerciaux », qui ne sont pas étudiés dans l'ouvrage ? À quel moment, avec quel studio, avec quelle équipe, les réalisateurs controversés sont-ils admis à faire un film ? Autant de questions qui ne sauraient retirer à cet ouvrage le mérite d'offrir un portrait détaillé de réalisateurs dont la carrière artistique a souffert de la censure et de mettre en évidence la portée et les effets, sur le long terme, de leur vision artistique du monde.

\section{NOTES}

1. Valerij Fomin, Polka: dokumenty, svidetel'stva, kommentarii [L'Étagère: documents, témoignages, commentaires], M. : Materik, 2006.

2. Josephine Woll, Real Images: Soviet Cinema and the Thaw, Londres: Tauris, 2000 ; Aleksandr Prohorov, Unasledovannyi diskurs: Paradigmy stalinskoi kul'tury $v$ literature $i$ kinematografe "ottepeli» [Le discours hérité : paradigmes de la culture stalinienne dans la littérature et le cinéma du « dégel »], SPb. : Akademicheskij proekt, 2007 ; Martin Dewhirst, Robert Farrell, éds., The Soviet Censorship, Metuchen NJ : The Scarecrow Press, 1973. 